\title{
Editorial: Living and Editing in the Online World
}

We are now enduring the second year of the Coronavirus pandemic. It has undoubtedly left its mark on the lives and well-being of many. Likewise, the pandemic has probably permanently changed our working habits too. It is not far-fetched to claim that even after the pandemic subsides we will be working more from our homes and long distance. Most of us have now finally had to make the long and tumultuous leap to the online world and the realm of digital humanities.

While this transition has undoubtedly been a strain, it is also a possibility. For example, I recently listened to a lecture organised by a Scottish university, given by a speaker in the USA, in the comfort of my kitchen. And while circumstances made it impossible to spend a few months as a visiting scholar in Cambridge, UK, I have nevertheless been able participate in a reading group on a weekly basis. Something like this would have been unthinkable earlier.

This new situation gives us a pause to consider how much and often we ought to travel to conferences and other universities for visits in the future. I think that mobility and meetings in person will be important also in the years to come for the dissemination of ideas and for acquiring understanding of other peoples, cultures and locations. Phenomenologically, it is quite different to be in a place than be connected to it through a screen. Tastes, smells, temperatures, movements, non-verbal and informal communication are all or mostly missed in onscreen meetings. Nevertheless, the new situation requires more careful thinking regarding what we should in fact do when we go to a foreign place to visit. Travelling is no longer a prerequisite for listening to talks and lectures abroad. Perhaps we have entered the era of less frequent but more profound and intensive visits and collaborations.

The Corona pandemic and our newly acquired digital skills are also bound to affect how journals are edited. I have previously thought that unquestionably the best model for editing involves people physically meeting in the same room. Although this would still be desirable, I am now less sure about how necessary and advantageous it is. So much, including meetings, can be done online. This long distance form of editing has in fact been the model of Journal of Philosophy of History and is likely to continue.

Fortunately, our writers and readers online have been active before and during the pandemic, and in spite of it. There has been a steady increase in 
downloads of papers in 2015-2019, the latest figure being above 12 ooo annual downloads. Further, whereas previously we had a backlog of accepted but not yet printed articles, this problem has now been largely sorted out. One consequence is that our acceptance rate has shrunk to $20 \%$ of unsolicited submissions. This means that all our authors can take pride in publishing their research in Journal of the Philosophy of History. They can be sure that the papers have been rigorously vetted.

In this issue, Alison M. Downham Moore focuses on the challenges of writing long conceptual histories, and specifically in the field of sexual medicine, in her paper "Temporal Layering in the Long Conceptual History of Sexual Medicine: Reading Koselleck with Foucault." She emphasises the need to develop further conceptual methodologies, and shows how both Koselleck and Foucault are useful guides in this endeavour. The issue of continuity is also in the heart of Andrew Fiala's "Progress and Meliorism: Making Progress in Thinking about Progress." His focus is on the notions of progress and meliorism. Fiala argues that meliorism is epistemically modest in that it commits only to the idea of local and incremental improvement, and without an aim of perfection. He argues that we have made progress historically in that our understanding has deepened: we have learnt from our past debates about progress and the Enlightenment.

After these conceptual examinations and forays into the problems of conceptual history, the issue moves on to the investigation of three different theory traditions in philosophy. In his "Can Normic Laws Save Hempel's Model of Historical Explanation? A Critique of Schurz' Approach," Gunnar Schuman takes a fresh angle on the old debate between causalists and teleologists about explanation. He critically assesses Gerhard Schurz' attempt to develop Hempel's covering law model to fit human action in the historical sciences. Schuman argues that there is a conceptual connection between volition and action, and further, that the methods of explanation in science and historiography are crucially different. Next, Jonathan Menezes examines Frank Ankersmit's postmodernism in his "The Limits of the 'Autumn of Historiography': On Frank Ankersmit's Postmodernist Moment." Menezes shows that Ankersmit experienced a 'postmodernist moment' at a time when postmodernism was almost "the only game in town in theory history," and that while Ankersmit initially developed and defended postmodernism, subsequently, he turned against it arguing for its irresponsibility. Finally, Adam Bricker evaluates the recently emerged postnarrativism in philosophy of history. According to Bricker, the postnarrativist option to divorce justification from truth with regard to historiographically interesting statements, or comprehensive "content-synthesizing entities," is problematic. He writes that these 
problems can be solved by thinking that all claims are either true or false, and furthermore, that postnarrativism is, in actuality, amenable to this kind of binary truth-functional thinking.

In addition, there are two book reviews in this issue. Jonas Ahlskog reviews The Ethos of History: Time and Responsibility, which is edited by Stefan Helgesson and Jayne Svenungsson. Aviezer Tucker assesses Adrian Currie's Rock, Bone, and Ruin: An Optimist's Guide to the Historical Sciences.

I am sure that I speak for all the editors in expressing our great satisfaction that we are able to again present an extremely stimulating issue with diversity and rigour that will be of interest to many. And I thank all our readers, writers and reviewers, and the copyeditors at the University of Oulu, for the past year. I wish you a good, and improving, continuing year of 2021.

Jouni-Matti Kuukkanen

Editor-in-Chief

Journal of the Philosophy of History 